\title{
Effective Hamiltonian for nickelate oxides $\mathrm{Nd}_{1-x} \mathrm{Sr}_{x} \mathrm{NiO}_{2}$
}

\author{
Hu Zhang, ${ }^{1}$ Lipeng Jin, ${ }^{1}$ Shanmin Wang, ${ }^{1}$ Bin Xi, ${ }^{2, *}$ Xingqiang Shi $\odot,{ }^{1,}{ }^{\dagger}$ Fei Ye, ${ }^{1}$ and Jia-Wei Mei ${ }^{1, \ddagger}$ \\ ${ }^{1}$ Shenzhen Institute for Quantum Science and Engineering and Department of Physics, \\ Southern University of Science and Technology, Shenzhen 518055, China \\ ${ }^{2}$ College of Physics Science and Technology, Yangzhou University, Yangzhou 225002, China
}

(Received 27 September 2019; revised manuscript received 2 December 2019; accepted 28 January 2020; published 26 February 2020)

\begin{abstract}
We derive the effective single-band Hamiltonian in the flat $\mathrm{NiO}_{2}$ planes for nickelate compounds $\mathrm{Nd}_{1-x} \mathrm{Sr}_{x} \mathrm{NiO}_{2}$. We implement the first-principles calculation to study electronic structures of nickelates using the Heyd-Scuseria-Ernzerhof hybrid density functional and derive a three-band Hubbard model for Ni-O $p d \sigma$ bands of $\mathrm{Ni}^{+} 3 d_{x^{2}-y^{2}}$ and $\mathrm{O}^{2-} 2 p_{x / y}$ orbitals in the $\mathrm{NiO}_{2}$ planes. To obtain the effective one-band $t-t^{\prime}-J$ model Hamiltonian, we perform the exact diagonalization of the three-band Hubbard model for the $\mathrm{Ni}_{5} \mathrm{O}_{16}$ cluster and map the low-energy spectra onto the effective one-band models. We find that the undoped $\mathrm{NiO}_{2}$ plane is a Hubbard Mott insulator and the doped holes are primarily located on $\mathrm{Ni}$ sites. The physics of the $\mathrm{NiO}_{2}$ plane is a doped Mott insulator, described by the one-band $t-t^{\prime}-J$ model with $t=265 \mathrm{meV}, t^{\prime}=-21 \mathrm{meV}$, and $J=28.6 \mathrm{meV}$. We also discuss the electronic structure for the self-doping effect and heavy fermion behavior of electron pockets of $\mathrm{Nd}^{3+} 5 d$ character in $\mathrm{Nd}_{1-x} \mathrm{Sr}_{x} \mathrm{NiO}_{2}$.
\end{abstract}

DOI: 10.1103/PhysRevResearch.2.013214

\section{INTRODUCTION}

The layered high-temperature superconductors in copper oxides and iron pnictides have motivated the search for new superconductivity compounds with layered structures [1-4]. Due to the similar crystal and electronic structure, $\mathrm{LaNiO}_{2}$ has been studied theoretically as a possible analog to the cuprates $[5,6]$. Recently, superconductivity with a critical temperature up to $T_{c} \sim 15 \mathrm{~K}$ has indeed been discovered in $\mathrm{Nd}_{0.8} \mathrm{Sr}_{0.2} \mathrm{NiO}_{2}$ thin film [7], although the presence of superconductivity remains debated [8,9]. Similar to copper oxides, perovskite nickelates $\left(R \mathrm{NiO}_{3}\right.$, where $R$ is a rare-earth or heavy metal such as $\mathrm{Tl}$ or $\mathrm{Bi}$ ) display many strongly correlated physical properties, such as sharp metal-insulator transitions, particular magnetic order, and charge order [10-13]. The reduced form of $\mathrm{RNiO}_{3}$ leads to the infinite layered phase $R \mathrm{NiO}_{2}$ [14-20], which has a very flat $\mathrm{NiO}_{2}$ plane of the square lattice for $\mathrm{Ni}^{+}$with one hole in the $d_{x^{2}-y^{2}}$ orbital. The superconductivity likely occurs in the $\mathrm{NiO}_{2}$ plane with the charged carrier doping. In the cuprate superconductor compounds, the strong electronic interactions play a significant role in the electronic structure [21], and the effective one-band Hamiltonian has been proposed to describe the low-energy physics of the correlation effects for $3 d^{9}$ electrons [21-23]. To understand the strongly correlated electronic structures of the nickelate

\footnotetext{
*xibin@yzu.edu.cn

†shixq@sustech.edu.cn

${ }^{\ddagger}$ meijw@ sustech.edu.cn
}

Published by the American Physical Society under the terms of the Creative Commons Attribution 4.0 International license. Further distribution of this work must maintain attribution to the author(s) and the published article's title, journal citation, and DOI. oxides $\mathrm{Nd}_{1-x} \mathrm{Sr}_{x} \mathrm{NiO}_{2}$, we need to find the proper effective (one-band) Hamiltonian to explore the similarity to and difference from the cuprate compounds.

$\mathrm{NdNiO}_{2}$ crystallizes in the $P 4 / \mathrm{mmm}$ (No. 123) space group, as depicted in Fig. 1(a). Four oxygens surround the nickel in a planar square environment [Fig. 1(d)] and the crystal field splits the $d$ orbitals as shown in Fig. 1(e). $\mathrm{Ni}^{+}$has the $d^{9}$ electronic state configuration and the highest partially occupied $d$ orbital is $3 d_{x_{2}-y_{2}}$. The rare-earth ion $\mathrm{Nd}^{3+}$ sits in the center of a cuboid formed by eight oxygen ions as shown in Fig. 1(c). Like $\mathrm{Nd}^{3+}$ in $\mathrm{Nd}_{2} \mathrm{CuO}_{4}$ [24] and $\mathrm{Ho}^{3+}$ in $\mathrm{HoNiO}_{3}$ [25], $\mathrm{Nd}^{3+}$ has the local $4 f$ moment far below the Fermi energy level. $\mathrm{Nd} 5 d$ orbitals have the split energy levels, as shown in Fig. 1(e), and near the Fermi energy, the lowest $5 d$ orbital in $\mathrm{Nd}^{3+}$ is $d_{z^{2}}$. Therefore, in the simple reckoning for the relevant electronic structure, there are 12 bands near the Fermi energy level corresponding to mainly $\mathrm{Ni} d$ (5 states), $\mathrm{O} p(2 \times 3$ states $)$, and $\mathrm{Nd} d_{z^{2}}$ (1 state). Previous density functional theory (DFT) studies within the local density approximation (LDA) on $\mathrm{LaNiO}_{2}$ [6] support the rough impression of the nonmagnetic electronic structures.

In this paper we first implement the first-principles simulations to derive a three-band Hubbard model for $\mathrm{Ni}-\mathrm{O} p d \sigma$ bands of $\mathrm{Ni}^{+} 3 d_{x^{2}-y^{2}}$ and $\mathrm{O}^{2-} 2 p_{x / y}$ orbitals in the $\mathrm{NiO}_{2}$ planes. Based on the three-band Hubbard model, we perform the exact diagonalization ${ }^{1}$ for the $\mathrm{Ni}_{5} \mathrm{O}_{16}$ cluster and obtain the low-energy one-band effective Hamiltonian for the $\mathrm{NiO}_{2}$ planes. We also discuss the electronic structure for the selfdoping effect and the heavy-fermion behavior of electron pockets of $\mathrm{Nd}^{3+} 5 d$ character in $\mathrm{Nd}_{1-x} \mathrm{Sr}_{x} \mathrm{NiO}_{2}$. We present our main results in two successive stages in Sec. II. We discuss

\footnotetext{
${ }^{1}$ The exact diagonalization is performed by the $\mathcal{H} \Phi$ package [26].
} 
(a)

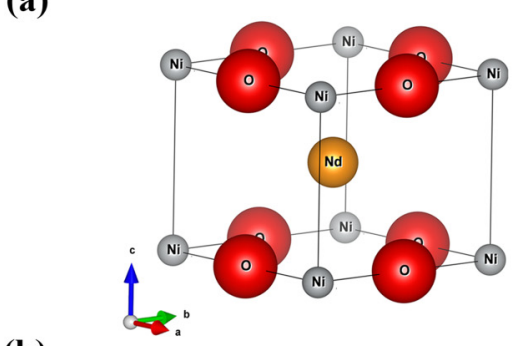

(b)

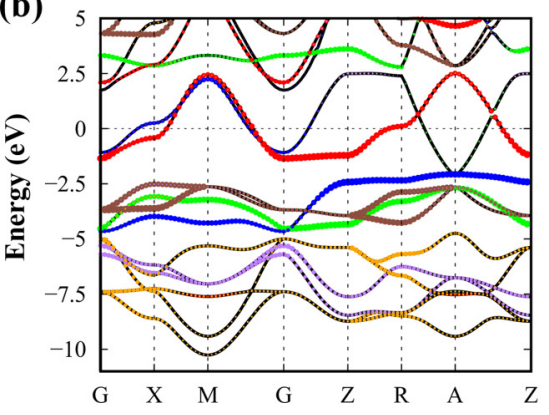

(c)

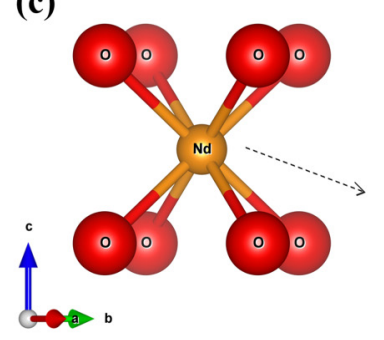

(d)

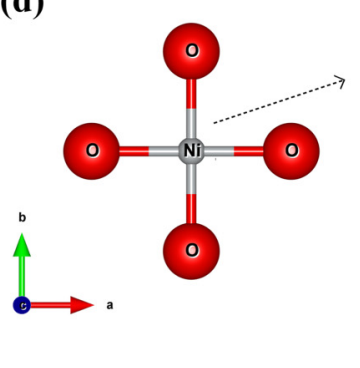

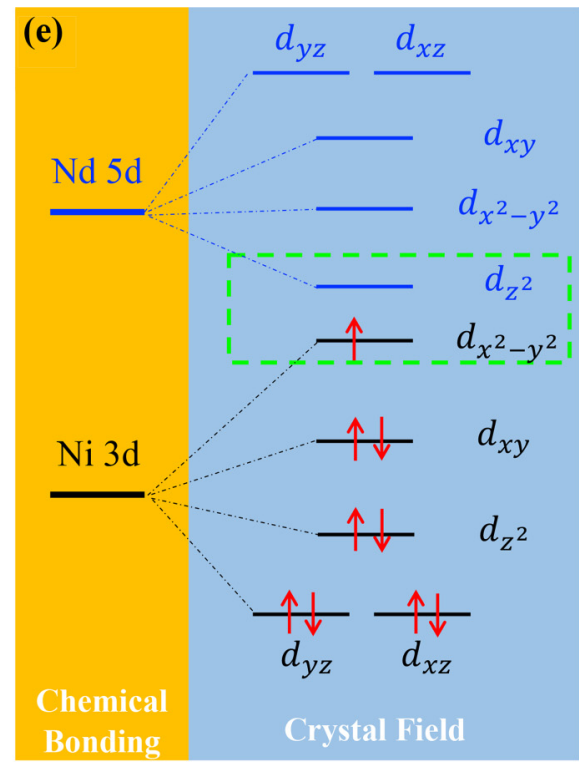

FIG. 1. (a) Crystal structure of $\mathrm{NdNiO}_{2}$ with space group P4/mmm (No. 123). (b) HSE06 band structures of the nonmagnetic state in the parent compound $\mathrm{NdNiO}_{2}$ along the $\Gamma(0,0,0)-X(0,0.5,0)-M(0.5,0.5,0)-\Gamma-Z(0,0,0.5)-R(0,0.5,0.5)-A(0.5,0.5,0.5) Z$ directions. The projected band structures of $d$ orbitals (red for $d_{x^{2}-y^{2}}$, blue for $d_{z^{2}}$, brown for $d_{x z / y z}$, and green for $d_{x y}$ ) in Ni and Nd and $2 p$ orbitals (orange for $p_{x / y}$ and purple for $p_{z}$ ) in $\mathrm{O}$ are also shown. The Fermi level is set at $0 \mathrm{eV}$. (c) $\mathrm{Nd}$ and its eight nearest-neighbor oxygens. (d) $\mathrm{Ni}$ and its four nearest-neighbor oxygens. (e) Crystal field splitting of $\mathrm{Nd} 5 d$ and $\mathrm{Ni} 3 d$ orbitals.

the physics of the one-band $t-J$ model in the $\mathrm{NiO}_{2}$ planes in Sec. III. We include additional results for $\mathrm{Nd}_{1-x} \mathrm{Sr}_{x} \mathrm{NiO}_{2}$ in Appendix A. In Appendix B we present the results for $\mathrm{La}_{1-x} \mathrm{Sr}_{x} \mathrm{NiO}_{2}$, implying the generic electronic structures of $R \mathrm{NiO}_{2}$ series.

The main results are summarized as follows. In Sec. II A we first perform DFT calculations of $\mathrm{Nd}_{1-x} \mathrm{Sr}_{x} \mathrm{NiO}_{2}$ within the Heyd-Scuseria-Ernzerhof (HSE) hybrid density functional. We note that in the previous study of the nickelates $\mathrm{RNiO}_{3}$, the HSE hybrid functional method was essential to reproduce the experimentally observed magnetic ground state [27]. On the generalized gradient approximation (GGA) level, DFT simulations suggest the $G$-type antiferromagnetic (AFM within the $\mathrm{NiO}_{2}$ plane and AFM between $\mathrm{NiO}_{2}$ planes along the $c$ direction) ground state of the moment on $\mathrm{Ni}$ sites in the parent compound $\mathrm{NdNiO}_{2}$. The HSE results for $\mathrm{Nd}_{1-x} \mathrm{Sr}_{x} \mathrm{NiO}_{2}$ adopt the $G$-type spin configuration to mimic strong spin correlations. In Sec. II B we describe the electronic structures based on the DFT simulations. Three-dimensional electron Fermi pockets of $\mathrm{Nd} 5 d$ character are found near the Fermi energy and behave as a heavy-fermion system due to its coupling with the local moments of $\mathrm{Nd} 4 f$, similar to the electron-doped cuprate $\mathrm{Nd}_{1.8} \mathrm{Ce}_{0.2} \mathrm{CuO}_{4}$ [28]. We also obtain the three-band Hubbard model for Ni-O $p d \sigma$ bands of $\mathrm{Ni}^{+} 3 d_{x^{2}-y^{2}}$ and $\mathrm{O}^{2-} 2 p_{x} / p_{y}$ orbitals in the $\mathrm{NiO}_{2}$ planes.

In Sec. IIC we derive the effective one-band Hamiltonian of the $\mathrm{NiO}_{2}$ plane, following the standard procedure in cuprates $[22,23]$. We derive the parameters for the three-band Hubbard model of the $\mathrm{Ni}-\mathrm{O} p d \sigma$ bands for $\mathrm{Ni}^{+} 3 d_{x^{2}-y^{2}}$ and $\mathrm{O}^{2-} 2 p_{x / y}$ orbitals from the LDA results from the first-principles simulations for the nonmagnetic ground state for $\mathrm{NdNiO}_{2}$. Exact diagonalization (ED) studies of the $\mathrm{Ni}_{5} \mathrm{O}_{16}$ cluster within the three-band Hubbard model are used to select and map the low-energy spectra onto the effective one-band $t-t^{\prime}-J$ model. According to ED results on finite clusters, in hole-doped nickelates, the doped holes are primarily located on $\mathrm{Ni}$ sites, in good agreement with the HSE results in Sec. II A and the experiment [29], while for cuprate the holes are mainly located on oxygen sites [22,23]. The physics of the $\mathrm{NiO}_{2}$ is a doped Mott insulator described by the effective one-band $t-t^{\prime}-J$ model with $t=265 \mathrm{meV}$, $t^{\prime}=-21 \mathrm{meV}$, and $J=28.6 \mathrm{meV}$. The one-band effective Hubbard model is also given.

\section{RESULTS}

\section{A. DFT results of $\mathrm{Nd}_{1-x} \mathrm{Sr}_{x} \mathrm{NiO}_{2}$}

We performed first-principles calculations based on DFT [30] with the HSE06 hybrid functional [31] as implemented in the Vienna Ab initio Simulation Package [32-34]. We also implement the Perdew-Burke-Ernzerhoff (PBE) functional in the GGA [35] and the strongly constrained and appropriately normed semilocal density functional (SCAN) in meta-GGA [36] for comparison. We use an energy cutoff of $500 \mathrm{eV}$ and $12 \times 12 \times 12,4 \times 4 \times 4$, and $4 \times 4 \times 4$ Monkhorst-Pack grids [37] in the PBE, SCAN, and HSE06 calculations, respectively. The $4 f$ orbitals in $\mathrm{Nd}^{3+}$ are expected to display the local magnetic moment as $\mathrm{Nd}^{3+}$ in $\mathrm{Nd}_{2} \mathrm{CuO}_{4}$ [24] and $\mathrm{Ho}^{3+}$ in $\mathrm{HoNiO}_{3}$ [25], and we treat them as the core-level electrons in the Nd pseudopotential in the HSE06 calculations. We check the results with the $4 f$ valence electron pseudopotential within the GGA $+U\left(U_{4 f}=10 \mathrm{eV}\right)$ scheme [38] in Appendix A. We also check that the spin-orbit coupling (SOC) does not significantly change the DFT results in Appendix A. Therefore, we only present the simulations by taking $4 f$ the core-level electrons in the $\mathrm{Nd}$ pseudopotential and do not 
include the spin-orbit coupling in our HSE06 hybrid functional simulations.

We use the bulk lattice constants $a=b=3.9208 \AA$ and $c=3.281 \AA$ [16] for $\mathrm{Nd}_{1-x} \mathrm{Sr}_{x} \mathrm{NiO}_{2}$ and do not optimize the crystal structure during the DFT simulations. In this setup, the $\mathrm{NiO}_{2}$ plane remains flat in the doped case in $\mathrm{Nd}_{0.75} \mathrm{Sr}_{0.25} \mathrm{NiO}_{2}$. We do not consider the lattice distortion effect upon doping in the doped nickelate oxides.

Figure 1(b) is the HSE06 band structure for the nonmagnetic state in $\mathrm{NdNiO}_{2}$. The band ordering is different from the crystal field theory in Fig. 1(e) due to the covalent effect in the hybridizations between $d$ orbitals $(\mathrm{Nd}$ and $\mathrm{Ni}$ ) and $2 p$ orbitals $(\mathrm{O})$. There are 12 electronic bands near the Fermi energy $E_{F}$, corresponding to orbitals mainly of $\mathrm{Ni} d$ (5 states) and $\mathrm{O} p$ $(2 \times 3$ states $)$ and $\mathrm{Nd} d_{z^{2}}$ (1 state) for the relevant electronic structure. The $\mathrm{O} 2 p$ bands extend from about -10 to $-5 \mathrm{eV}$. The Ni $3 d$ bands are distributed from -5 to $2 \mathrm{eV}$, while the broad Nd $5 d$ states range from -1 to $10 \mathrm{eV}$. The Ni $3 d_{x^{2}-y^{2}}$ and $\mathrm{Nd} 5 d_{z^{2}}$ cross the Fermi energy $E_{F}$ as expected from the crystal field splitting, as shown in Fig. 1(e). Ni $3 d_{x^{2}-y^{2}}$ is very broad along the $\Gamma-X-M$ direction due to the strong $d p \sigma$ antibonding interaction with oxygen $p_{x / y}$ states and encloses holes centered at the $M$ point. Ni $3 d_{x y / x z / y z}$ bands localize near $-4 \mathrm{eV}$ due to the weak $d p \pi$ hybridization with $\mathrm{O} 2 p$ states. Around the $A$ point, the Nd $5 d_{z^{2}}$ state forms an electron pocket around the $\Gamma$ point, goes up along the $\Gamma-Z$ direction, and lies above the Fermi energy level with $k_{z}=\pi / c$. The $\mathrm{Nd} 5 d_{x y}$ state goes down and crosses the Fermi level, forming an electron pocket around the $A$ point.

Along the $\Gamma-Z$ direction, four $\mathrm{O} 2 p_{x / y}$ bands and four $\mathrm{Ni}$ $\left(3 d x z / y z, 3 d_{x y}\right.$, and $\left.3 d_{x^{2}-y^{2}}\right)$ bands have weak dispersions, indicating the two-dimensional features of these bands. Ni $3 d_{z^{2}}$ and $\mathrm{Nd} 5 d$ states are dispersive along the $\Gamma-Z$ directions and are three-dimensionally extended. The two electron pockets around the $\Gamma$ and $A$ points of the Nd $5 d$ orbital character have the Ni orbitals mixing. However, such mixing is not quickly resolved in Fig. 1(b) since the Nd $5 d$ characters dominate the electron pockets and cover up the contributions from $\mathrm{Ni}$ orbitals. To further clarify the mixing character, we also present the band structure for the simulated $\mathrm{SrNiO}_{2}$ with the same structure as $\mathrm{NdNiO}_{2}$, in order to eliminate the $\mathrm{Nd} 5 d$ orbitals in Fig. 2. From a comparison of Figs. 1(b) and 2 we can see that the Nd $5 d_{z^{2}}$ band crosses $E_{F}$ around the $\Gamma$ point with $\mathrm{Ni} 4 s$ mixing, while the $\mathrm{Nd} 5 d_{x y}$ band crosses $E_{F}$ around the $A$ point with $\mathrm{Ni} 4 p_{z}$ mixing.

The GGA band structure of $\mathrm{NdNiO}_{2}$ in Appendix A is quite similar to the LDA band structure of $\mathrm{LaNiO}_{2}$ [6], indicating a generic electronic structure in the $R \mathrm{NiO}_{2}$ family. Compared with the GGA band structure (Appendix A), for the nonmagnetic state, the mixing of the exact exchange in the HSE06 hybrid functional separates the two bands crossing the Fermi energy $E_{F}$ away from other bands, without significant change of dispersions and relative positions of the bands far from $E_{F}$. The separation of two bands can also be reproduced in the LDA $+U$ results [6]. However, in the LDA $+U$ scheme, Ni $3 d_{z^{2}}$ is raised by $U$ and crosses $E_{F}$ when $U$ is large [6], different from the HSE06 hybrid functional simulation for the nonmagnetic state.

The magnetization measurement and neutron powder diffraction did not reveal the long-magnetic order in $\mathrm{LaNiO}_{2}$
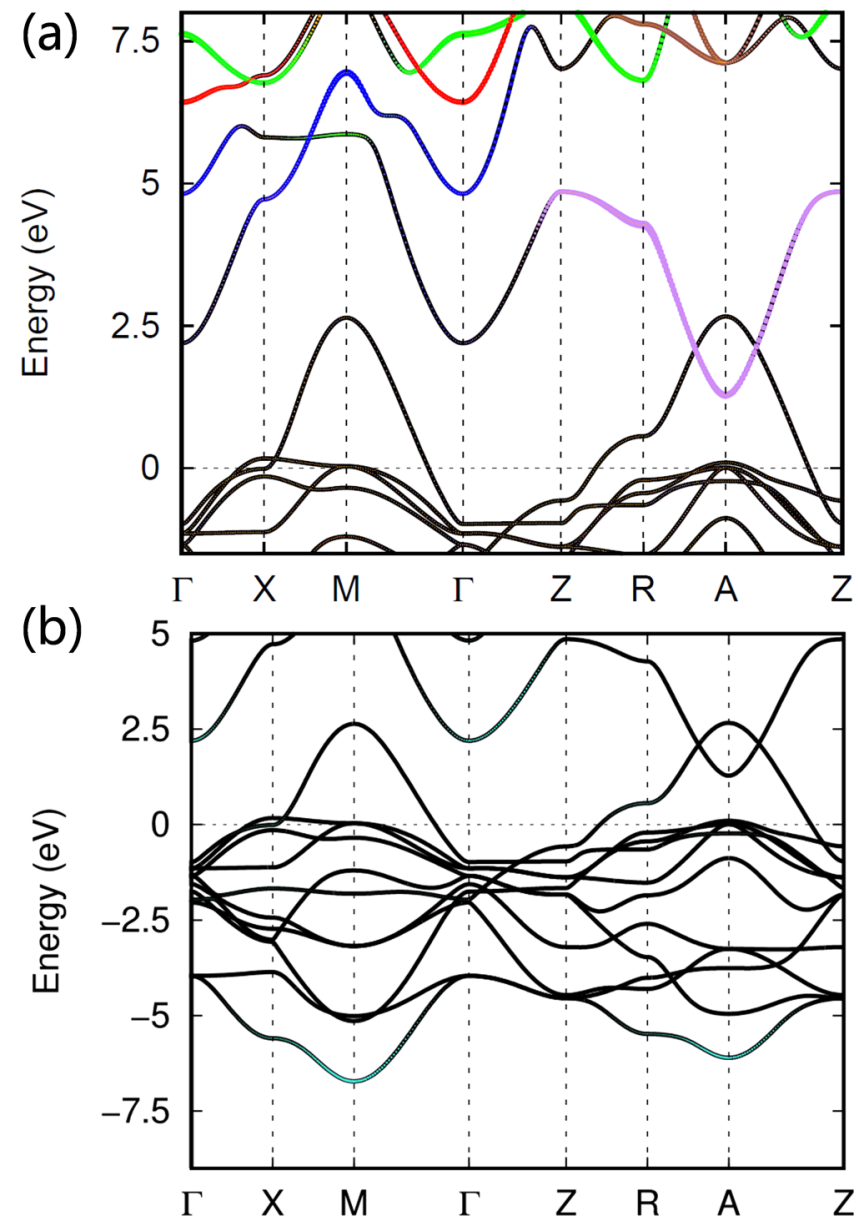

FIG. 2. GGA band structures of the nonmagnetic state in the simulated $\mathrm{SrNiO}_{2}$ with the same structure as $\mathrm{NdNiO}_{2}$. The projected band structures of $d$ orbitals in $\mathrm{Sr}$ and $4 p$ orbitals in $\mathrm{Ni}$ are also shown. The color in (a) has the same meanings as in Fig. 1(b). The color turquoise in (b) indicates the projected Ni $4 s$ orbital (mainly located at the $\Gamma$ point at around $2.4 \mathrm{eV}$ ).

and $\mathrm{NdNiO}_{2}$; however, the paramagnetic susceptibilities imply the (at least short) spin correlations $[15,16]$. The absence of long-range magnetic order may be due to poor sample qualities or due to the self-doping effects of the $\mathrm{Nd} 5 d$ electron pockets. The strong correlation for electrons on $\mathrm{Ni}^{+}$induce the magnetism in the system.

To demonstrate the correlation and magnetism in $\mathrm{NdNiO}_{2}$, we calculate the magnetic moment and compare the ground state with the nonmagnetic one within different functionals (GGA, SCAN, and HSE06). To save computation time, we consider the ferromagnetic spin configuration in the primary unit cell. The results are listed in Table I. Compared with the nonmagnetic state, the magnetic states have increased moments on $\mathrm{Ni}$ with increasingly lower ground-state energies from the GGA to SCAN to HSE06. For the GGA, the magnetic state has even higher energy than the nonmagnetic state, indicating the nonmagnetic ground state within the GGA functional, consistent with the previous study on $\mathrm{LaNiO}_{2}$ [6]. The SCAN functional includes more correlation effects and the magnetism is significantly enhanced. The magnetic ground state has even lower energy than the nonmagnetic state in the HSE06 functional. The fact that the correlation achieves 
TABLE I. Theoretical magnetic moments $\left(\right.$ in $\mu_{B}$ ) on $\mathrm{Ni}$ in $\mathrm{NdNiO}_{2}$ calculated with the HSE06, SCAN, and PBE functionals. Here $\Delta E$ is the energy difference between the nonmagnetic state and the ferromagnetic state.

\begin{tabular}{lcc}
\hline \hline Functional & Magnetization & $\Delta E(\mathrm{meV})$ \\
\hline HSE06 & 0.94 & 744.7 \\
SCAN & 0.76 & 142.7 \\
GGA & 0.05 & -0.1 \\
\hline
\end{tabular}

the magnetism is a strong indication that $\mathrm{NdNiO}_{2}$ is magnetic if we include the correlations.

Even though there is no long-range magnetic order, we still impose static magnetic configurations on $\mathrm{Ni}$ ions in $\mathrm{Nd}_{1-x} \mathrm{Sr}_{x} \mathrm{NiO}_{2}$ to mimic the spin correlations in the DFT simulations for electronic structures. We find that the $G$-type AFM state has the lowest ground-state energy within the GGA DFT simulations. Therefore, in the HSE06 simulations, we adopt the $G$-type AFM spin configurations on $\mathrm{Ni}$ using the $\sqrt{2} \times \sqrt{2} \times 2$ supercell to study the electronic structures of $\mathrm{Nd}_{1-x} \mathrm{Sr}_{x} \mathrm{NiO}_{2}(x=0,0.25)$. Therefore, the Brillouin zone is folded by the $G$-type AFM spin configuration. However, without any confusion, we still use the notation $\Gamma, X, M, Z$, $R$, and $A$ for high-symmetry $k$ points in the folded Brillouin zone.

Figure 3 displays the HSE06 band structures of $\mathrm{Nd}_{1-x} \mathrm{Sr}_{x} \mathrm{NiO}_{2}(x=0,0.25)$ with the $G$-type AFM configuration on $\mathrm{Ni}$. In the HSE06 hybrid functional results, the AFM configuration not only folds the band structure, but also dramatically changes the bands near the Fermi energy $E_{F}$, very different from the GGA result for the $G$-type AFM configuration in Appendix A. The significant change of band structures in the AFM states between GGA and HSE06 implies strong correlations in $\mathrm{Nd}_{1-x} \mathrm{Sr}_{x} \mathrm{NiO}_{2}$.

In the $G$-type AFM state of $\mathrm{NdNiO}_{2}$ [Fig. 3(a)], the $\mathrm{Nd} 5 d_{z^{2}}$ band is raised above the Fermi energy $E_{F}$ by the correlation, eliminating the electron pocket of the $\mathrm{Nd} 5 d_{z^{2}}$ character in the nonmagnetic state [Fig. 1(b)]. The $\mathrm{Nd} 5 d_{x y}$ band is also raised, but the electron pocket of this band still exists. We recall here again that the electron pocket of the $\mathrm{Nd} 5 d_{x y}$ character has the $\mathrm{Ni} 4 p_{z}$ orbital mixing. The $\mathrm{Nd} 5 d_{x y}$ electron pocket is located around the $\Gamma$ point in the folded magnetic Brillouin zone. Therefore, the AFM spin correlation has an essential influence on the self-doping effect of Nd $5 d$ electron pockets. For the Ni $3 d$ band, the AFM correlation significantly renormalizes the bandwidth. The Ni $3 d_{x^{2}-y^{2}}$ bands split into upper and lower Hubbard bands separated by around $5 \mathrm{eV}$ due to the AFM spin configuration, indicating the strong correlation in $\mathrm{NdNiO}_{2}$. The lower Hubbard $3 d_{x^{2}-y^{2}}$ band is located lower than the Ni $3 d_{z^{2}}$ band, leading the doping problem into a complicated situation.

When one $\mathrm{Sr}^{2+}$ ion substitutes $\mathrm{Nd}^{3+}$ in $\mathrm{NdNiO}_{2}$, we dope an extra hole into the $\mathrm{NiO}_{2}$ planes. To simulate the doped nickelate oxide, we calculate the band structures of $\mathrm{Nd}_{0.75} \mathrm{Sr}_{0.25} \mathrm{NiO}_{2}\left(\mathrm{Nd}_{3} \mathrm{SrNi}_{4} \mathrm{O}_{8}\right)$ in the $\sqrt{2} \times \sqrt{2} \times 2$ supercell with the $G$-type AFM spin configuration on $\mathrm{Ni}$ ions. Figure 3(b) is the HSE06 band structure of $\mathrm{Nd}_{0.75} \mathrm{Sr}_{0.25} \mathrm{NiO}_{2}$. The Nd $5 d_{x y}$ band goes up above the Fermi energy level and the band minimum is located at $0.2 \mathrm{eV}$. We can expect
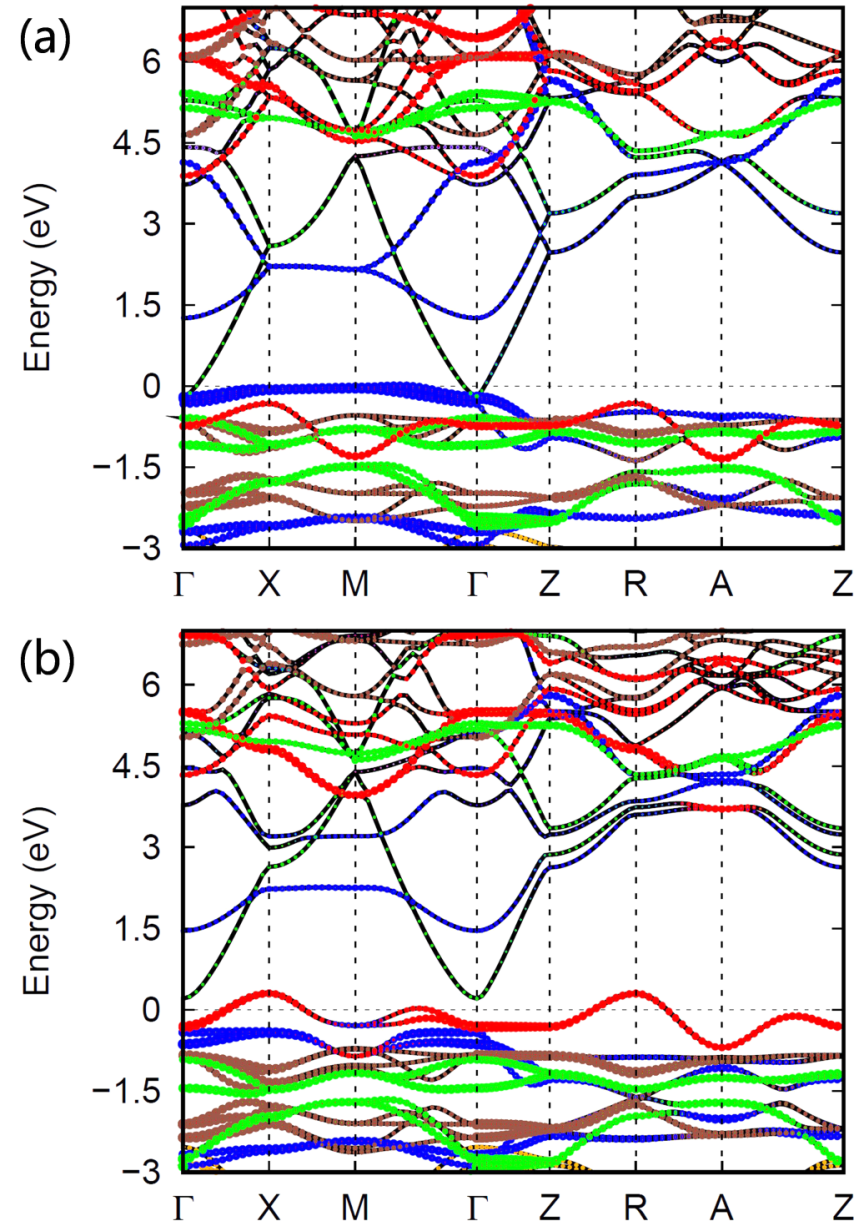

FIG. 3. HSE06 band structures of $G$-type AFM states for a $\sqrt{2} \times \sqrt{2} \times 2$ supercell in (a) $\mathrm{NdNiO}_{2}$ and (b) $\mathrm{Nd}_{0.75} \mathrm{Sr}_{0.25} \mathrm{NiO}_{2}$. The notation $\Gamma, X, M, Z, R$, and $A$ is in the folded magnetic Brillouin zone. The color has the same meanings as in Fig. 1(b).

that the $\mathrm{Nd} 5 d_{x y}$ band contributes a Hall coefficient at high temperatures at low dopings [7]. The doped hole does not go to the Ni $3 d_{z^{2}}$ band. Instead, it is located on the $3 d_{x^{2}-y^{2}}$ band, which goes up above $3 d_{z^{2}}$ in $\mathrm{Nd}_{0.75} \mathrm{Sr}_{0.25} \mathrm{NiO}_{2}$. Therefore, the doped hole does not polarize $\mathrm{Ni}^{2+}$ into the $S=1$ local moment, but creates an $S=0$ holelike doped charge carrier. The Ni $3 d_{x^{2}-y^{2}}$ encloses the hole pockets around the $X$ point in the folded Brillouin zone ( $M$ in the original nonmagnetic Brillouin zone). The HSE06 band structures in Fig. 3 suggest that the undoped $\mathrm{NiO}_{2}$ plane is a Hubbard-like Mott insulator, not the charge-transfer-like one as in the cuprate. The doped hole goes into the Ni $3 d_{x^{2}-y^{2}}$ orbital, creating an $S=0$ holelike charge carrier, rather than into the $\mathrm{O}^{2-} 2 p_{x / y}$ orbitals.

\section{B. Electronic structures of $\mathrm{Nd}_{1-x} \mathrm{Sr}_{x} \mathrm{NiO}_{2}$}

The presence of the electron pockets of $\mathrm{Nd} 5 d$ bands suggests the self-doping effect, implying a small charge transfer from these pockets to the Ni-O sheets even without chemical doping. The self-doping effect also exists in the cuprate family, e.g., $\mathrm{YBa}_{2} \mathrm{Cu}_{3} \mathrm{O}_{7}$ and $\mathrm{Bi}_{2} \mathrm{Sr}_{2} \mathrm{CaCu}_{2} \mathrm{O}_{8}$ [39]. The three-dimensional electron pockets of $\mathrm{Nd} 5 d_{x y}$ states have the mixing with $\mathrm{Ni} 4 p_{z}$ orbitals. The self-doping effect allows some charge transfer and changes the hole count in the 


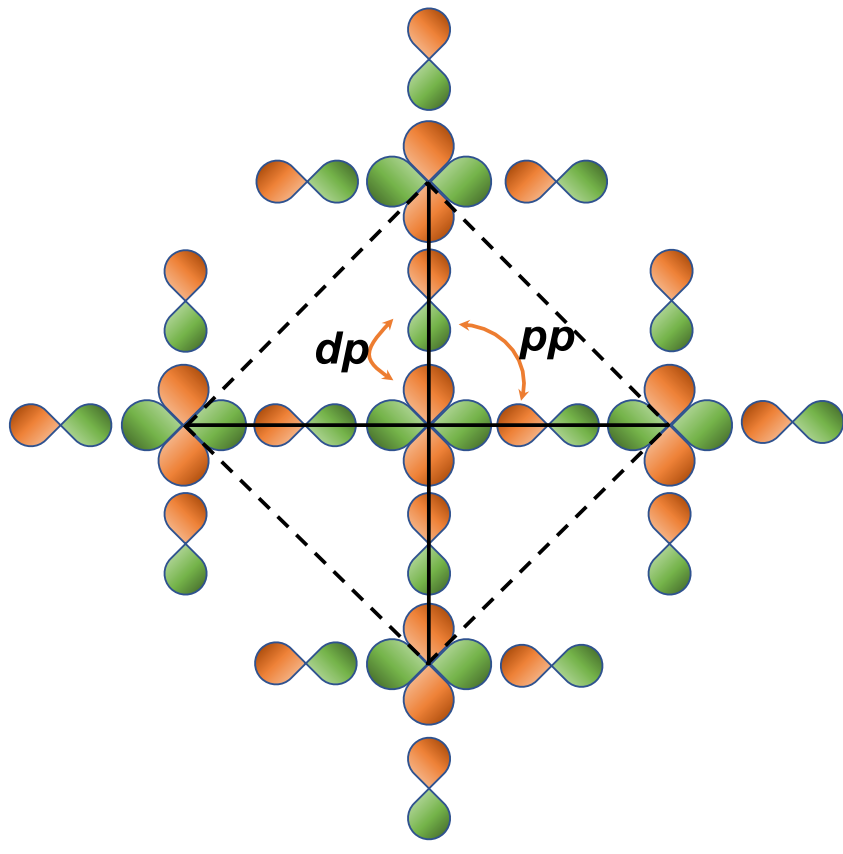

FIG. 4. Schematic representation of the orbitals (Ni $3 d_{x^{2}-y^{2}}$ and $\mathrm{O} p_{x / y}$ ) included in the three-band Hubbard model in the $\mathrm{Ni}_{5} \mathrm{O}_{16}$ cluster. The effective one-band model has the degree of freedom only on the five Ni $3 d_{x^{2}-y^{2}}$ orbitals connected by the solid ( $t$ and $J$ ) and dashed $\left(t^{\prime}\right.$ and $\left.J^{\prime}\right)$ lines.

$\mathrm{NiO}_{2}$ planes, resulting in the metallic behavior even without chemical doping. In Appendix A we present the GGA band structure with the $4 f$ valence electron pseudopotential within the GGA $+U$ scheme. We can see that $\mathrm{Nd} 4 f$ states have mixing with $\mathrm{Nd} 5 d$ states. Due to the presence of $\mathrm{Nd} 4 f$ orbitals, the electron pockets of the $\mathrm{Nd} 5 d_{x y}$ character hybridize with the $4 f$ local moments, behaving as a heavy-fermion system

$$
H_{K}=\sum_{\mathbf{k}} \epsilon_{\mathbf{k}} a_{\mathbf{k} \sigma}^{\dagger} a_{\mathbf{k} \sigma}+J_{K} \sum_{i} \mathbf{s}_{i} \cdot \mathbf{S}_{i}^{4 f},
$$

where $a_{\mathbf{k} \sigma}$ creates a fermion with momentum $\mathbf{k}$ on the electron pockets and $\mathbf{s}_{i}$ and $\mathbf{S}_{i}^{4 f}$ are the spin operators for the $5 d$ electrons and $4 f$ local moments, respectively. The interaction $J_{k}$ was further justified in Ref. [40] with $J_{k} \simeq-0.5 \mathrm{eV}$ (ferromagnetic, i.e., anti-Kondo in sign).

We now turn to the physics of the $\mathrm{NiO}_{2}$ plane with the hole doping, following the process for the $\mathrm{CuO}_{2}$ planes in cuprates $[22,23]$. The HSE06 band structures in Fig. 3 suggest that the undoped $\mathrm{NiO}_{2}$ plane is a Hubbard-like Mott insulator and the doped hole goes into the Ni $3 d_{x^{2}-y^{2}}$ orbital, creating an $S=0$ holelike charge carrier. Therefore, the physics of the $\mathrm{NiO}_{2}$ plane with the charge doping is described by the three-band Hubbard model for the $d p \sigma$ bands of Ni $3 d_{x^{2}-y^{2}}$ and $\mathrm{O} 2 p_{x / y}$ orbitals. Figure 4 schematically presents the orbitals of $\mathrm{Ni} 3 d_{x^{2}-y^{2}}$ and $\mathrm{O} 2 p_{x / y}$ with the green and brown colors corresponding to the positive and negative signs of wave functions, respectively.

We assume a vacuum state $d^{10} p^{6}$ and introduce the operators $d_{i \sigma}^{\dagger}$ and $p_{l \sigma}^{\dagger}$ creating the $\mathrm{Ni} 3 d_{x^{2}-y^{2}}$ hole and the O $2 p_{x / y}$ hole at the $i$ th $\mathrm{Ni}$ site and the $l$ th $\mathrm{O}$ site, respectively, with the spin $\sigma=\uparrow / \downarrow$. The holes hop between Ni $3 d_{x^{2}-y^{2}}$ and $\mathrm{O} 2 p_{x / y}$ orbitals with amplitude $t_{d p}$ and among $\mathrm{O} 2 p_{x / y}$ orbitals with the amplitude $t_{p p}$. We set the on-site potential of Ni $3 d_{x^{2}-y^{2}}$ as $\epsilon_{d}=0$ and the chemical potential difference between $\mathrm{Ni} 3 d_{x^{2}-y^{2}}$ and $\mathrm{O} 2 p_{x / y}$ orbitals as $\epsilon=\epsilon_{p}-\epsilon_{d}$. The strong correlations involve the on-site interactions $U_{p}$ and $U_{d}$ and intersite interactions $U_{d p}$ and $U_{p p}$ for holes on $\mathrm{O} 2 p_{x / y}$ and Ni $3 d_{x^{2}-y^{2}}$ orbitals. The three-band Hubbard model of the $d p \sigma$ bands reads

$$
\begin{aligned}
H_{d p}= & \sum_{\langle i l\rangle \sigma} t_{d p}\left(d_{i \sigma}^{\dagger} p_{l \sigma}+\text { H.c. }\right)+\sum_{\left\langle l l^{\prime}\right\rangle \sigma} t_{p p}\left(p_{l \sigma}^{\dagger} p_{l^{\prime} \sigma}+\text { H.c. }\right) \\
& +\sum_{l \sigma} \epsilon p_{l \sigma}^{\dagger} p_{l \sigma}+U_{d} \sum_{i} n_{d i \uparrow} n_{d i \downarrow}+U_{p} \sum_{l} n_{l \uparrow} n_{l \downarrow} \\
& +\sum_{\langle i l\rangle} U_{d p} n_{d i} n_{p l}+\sum_{\left\langle l l^{\prime}\right\rangle} U_{p p} n_{p l} n_{p l^{\prime}}
\end{aligned}
$$

Here $\langle\cdots\rangle$ denotes the nearest-neighbor bonds.

\section{Effective $t-t^{\prime}-J$ model Hamiltonian of $\mathrm{NiO}_{2}$ planes}

The tight-binding parameters $t_{d p}, t_{p p}$, and $\epsilon$ can be obtained from the Wannier fitting $[41,42]$ of the 12 bands in the LDA simulations for the nonmagnetic band structure of $\mathrm{NdNiO}_{2}$. It is noteworthy that the HSE06 band structure in Fig. 1(b) contains the renormalization effect due to the strong correlations. There is double counting for the correlations if we obtain the tight-binding parameters from the HSE06 band structure. The parameters are given as $\epsilon=4.2 \mathrm{eV}, t_{p d}=1.3 \mathrm{eV}$, and $t_{p p}=0.6 \mathrm{eV}$. We choose a proper basis of $3 d_{x^{2}-y^{2}}$ and $2 p_{x / y}$ for all positive $t_{p d}$ and $t_{p p}$. To match the similar sizable band splitting of the lower and upper Hubbard bands of the Ni $3 d_{x^{2}-y^{2}}$ orbitals in the HSE06 band structure in Fig. 3, we set the interaction terms as $U_{d}=7.5 \mathrm{eV}, U_{p}=5.0 \mathrm{eV}$, $U_{p d}=4.0 \mathrm{eV}$, and $U_{p p}=2.0 \mathrm{eV}$ in the ED calculation for the derivation of the effective one-band Hamiltonian.

We follow the process in the cuprates [22,23] to obtain the effective one-band Hamiltonian of the $\mathrm{NiO}_{2}$ planes. We perform direct ED studies of the three-band Hubbard model $H_{d p}$ in Eq. (2) and find the effective one-band Hamiltonian by the low-energy spectrum mapping. We carry out the ED calculation for the $\mathrm{Ni}_{5} \mathrm{O}_{16}$ cluster as shown in Fig. 4 with five and six holes for the undoped and hole-doped $\mathrm{NiO}_{2}$ planes, respectively. The $\mathrm{Ni}_{5} \mathrm{O}_{16}$ cluster is embedded in an array of $\mathrm{Ni} 3 d^{9}$ sites which shift the effective on-site energy of the outer $\mathrm{O}$ orbitals due to the intersite Coulomb energy [23].

Figure 5 is the low-energy spectrum mapping for the $\mathrm{Ni}_{5} \mathrm{O}_{16}$ cluster with five holes in the insulating ground state of the undoped $\mathrm{NiO}_{2}$ planes. The spin-1/2 Heisenberg model

$$
H_{J}=\sum_{i j} J_{i j} \mathbf{S}_{i} \cdot \mathbf{S}_{j},
$$

with the nearest-neighbor and next-nearest-neighbor exchange interactions $J=28.6 \mathrm{meV}$ and $J^{\prime}=0.4 \mathrm{meV}$, reproduces well the low-energy spectrum for the three-band Hubbard model as shown in Fig. 5. Therefore, we obtain the effective Heisenberg model for the undoped $\mathrm{NiO}_{2}$ planes.

For the hole-doped phase, the calculated low-energy spectra for six holes in the $\mathrm{Ni}_{5} \mathrm{O}_{16}$ cluster is shown in Fig. 6. The chemical character of the hole in the ground state of the doped $\mathrm{NiO}_{2}$ planes is of $\mathrm{Ni}(76 \%)$ and $\mathrm{O}(24 \%)$ character, indicating that $\mathrm{NdNiO}_{2}$ is in the regime of a Hubbard-Mott insulator, 


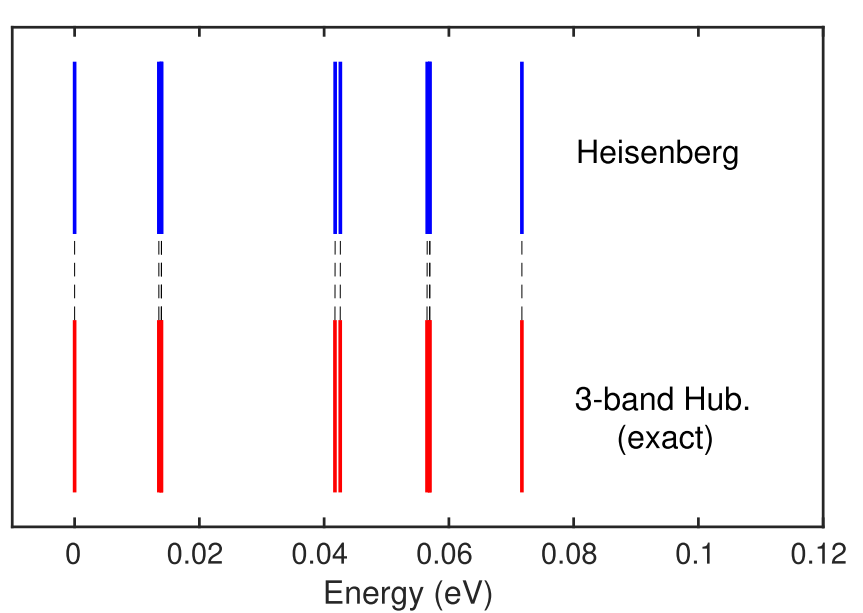

FIG. 5. Low-energy spectrum for the $\mathrm{Ni}_{5} \mathrm{O}_{16}$ cluster calculated in the three-band Hubbard model in comparison to the mappings onto the Heisenberg Hamiltonian for the five-hole case.

different from the cuprate where the doped holes are primarily located on $\mathrm{O}[22,23]$. The significant difference comes from a smaller $U_{d}$ but a larger $\epsilon=\epsilon_{p}-\epsilon_{d}$ in $\mathrm{NdNiO}_{2}$ than those in $\mathrm{La}_{2} \mathrm{CuO}_{4}$.

We map the three-band Hubbard model in Eq. (2) onto the single-band $t-t^{\prime}-J$ model

$$
H_{t J}=\sum_{i j \sigma} t_{i j} c_{i \sigma}^{\dagger} c_{j \sigma}+\sum_{i j} J_{i j} \mathbf{S}_{i} \cdot \mathbf{S}_{j},
$$

where $c_{i \sigma}$ is the electron operator on the $\mathrm{Ni}$ sites connected by the solid $(t$ and $J)$ and dashed $\left(t^{\prime}\right.$ and $\left.J^{\prime}\right)$ lines in Fig. 4. We first set $J=28.6 \mathrm{meV}$ and $J^{\prime}=0.4 \mathrm{meV}$ as in the undoped case and then tune the hopping parameters $t$ and $t^{\prime}$. Suitable values for $t=265 \mathrm{meV}$ and $t^{\prime}=-21 \mathrm{meV}$ with the mapping spectra are shown in Fig. 6. The signs for $t$ and $t^{\prime}$ refer to hole notation. We note that the low-energy spectrum has a width around $58 \mathrm{meV}$, approximately twice that of the exchange strength $J=28.6 \mathrm{meV}$, in good agreement with the $t-J$ model calculations for the cuprate, which show that, independently of the value of $t$, the dressing of the hole moving in the antiferromagnetic background reduces the quasiparticle bandwidth to a value twice that of $J$ [43].

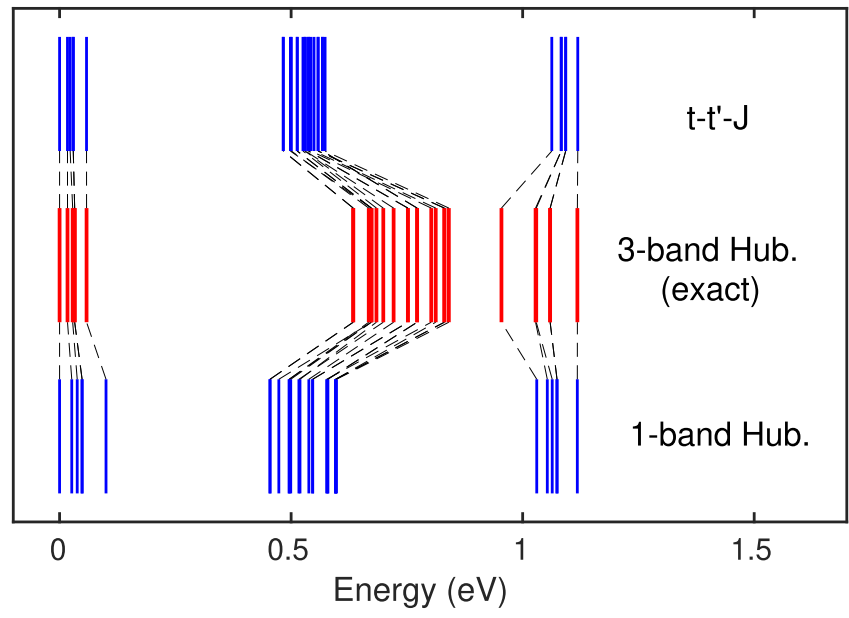

FIG. 6. Low-energy spectrum for $\mathrm{Ni}_{5} \mathrm{O}_{16}$ cluster mapping onto the effective one-band $t-t^{\prime}-J$ and Hubbard models.
We also map the low-energy spectra onto one-band Hubbard model

$$
H_{1 b}=\sum_{i j \sigma} t_{i j} c_{i \sigma}^{\dagger} c_{j \sigma}+U \sum_{i} n_{i \uparrow} n_{i \downarrow},
$$

with $t=254 \mathrm{meV}, t^{\prime}=-30 \mathrm{meV}$, and $U=6 \mathrm{eV}$ in Fig. 6 . The one-band Hubbard model gives a charge-transfer gap similar to that of the three-band Hubbard model in the $\mathrm{Ni}_{5} \mathrm{O}_{16}$ cluster.

\section{DISCUSSION AND CONCLUSION}

From the above derivation of the effective one-band $t-t^{\prime}-J$ model Hamiltonian, we find that the physics of $\mathrm{NiO}_{2}$ planes in $\mathrm{Nd}_{1-x} \mathrm{Sr}_{x} \mathrm{NiO}_{2}$ is a doped Mott insulator, even further away from a conventional Fermi liquid than the superconducting cuprates. The undoped $\mathrm{CuO}_{2}$ plane in the cuprate is a charge-transfer-type Mott insulator, but the undoped $\mathrm{NiO}_{2}$ plane in the nickelate is a Hubbard Mott insulator. Both the $\mathrm{NiO}_{2}$ and $\mathrm{CuO}_{2}$ planes have the same effective one-band
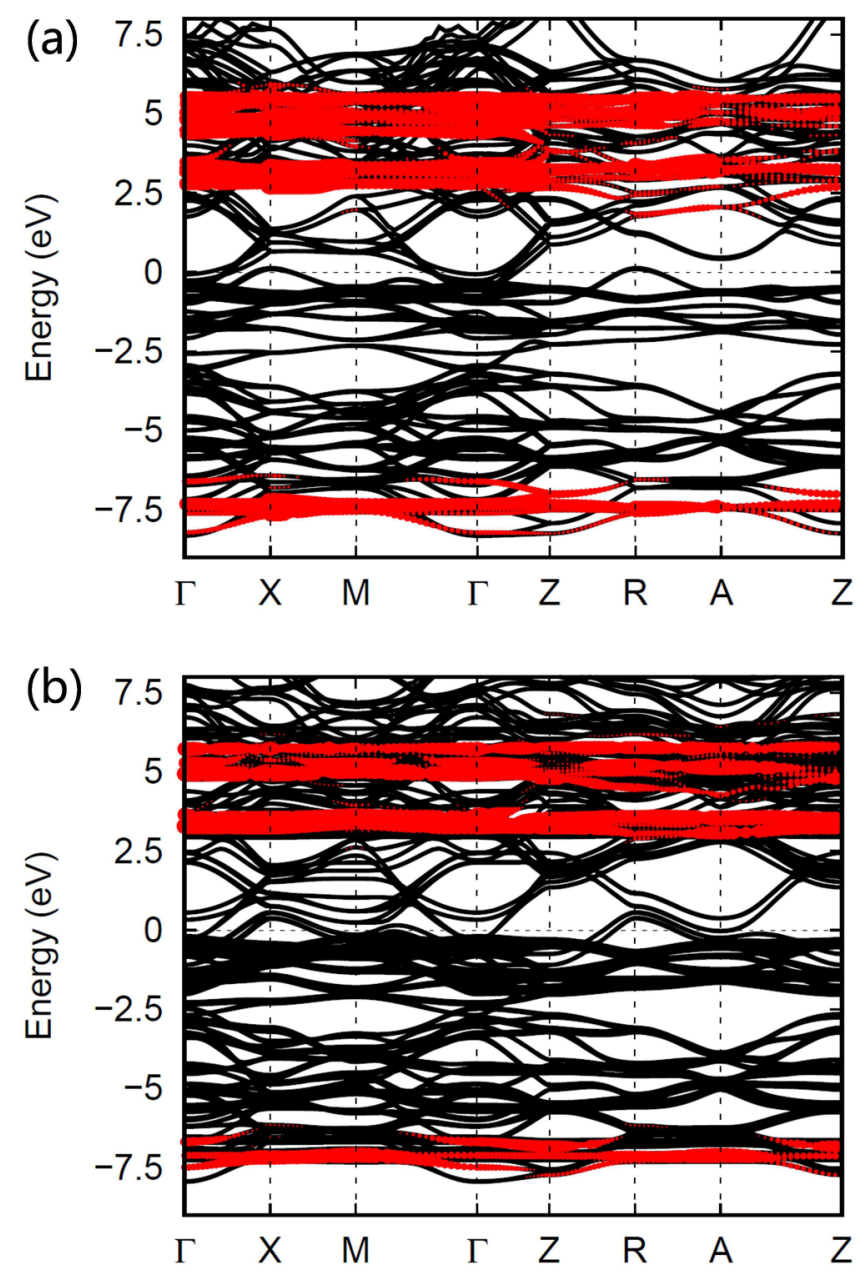

FIG. 7. GGA band structures of $G$-type AFM states for a supercell in (a) $\mathrm{NdNiO}_{2}$ and (b) $\mathrm{Nd}_{0.75} \mathrm{Sr}_{0.25} \mathrm{NiO}_{2}$ calculated with the $4 f$ valence electron pseudopotential within the GGA $+\mathrm{U}\left(U_{4 f}=\right.$ $10 \mathrm{eV}$ ) scheme with SOC. The notation $\Gamma, X, M, Z, R$, and $A$ is in the folded magnetic Brillouin zone. The projected band structures of $f$ orbitals (red) in Nd are also shown. The Fermi level is set at $0 \mathrm{eV}$. 

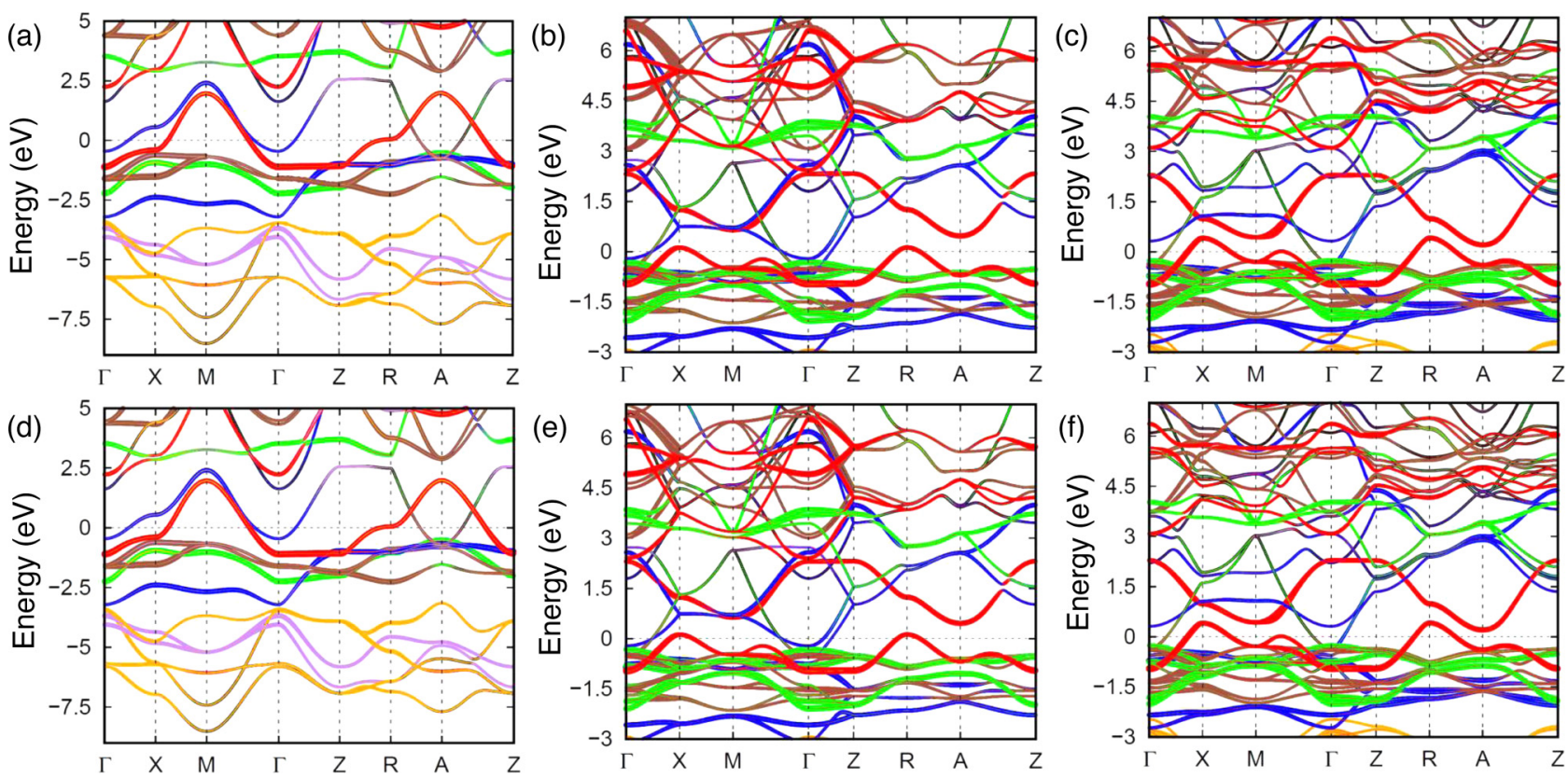

FIG. 8. GGA band structures of $\mathrm{Nd}_{1-x} \mathrm{Sr}_{x} \mathrm{NiO}_{2}$, corresponding to (a) Fig. 1(b) (the nonmagnetic state for $\mathrm{NdNiO}_{2}$ ), (b) Fig. 3(a) (the $G$-type AFM state for $\mathrm{NdNiO}_{2}$ ), and (c) Fig. 3(b) (the $G$-type AFM state for $\mathrm{Nd}_{0.75} \mathrm{Sr}_{0.25} \mathrm{NiO}_{2}$ ), without $\mathrm{SOC}$ and (d), (e), and (f), respectively, with SOC.

$t-t^{\prime}-J$ model Hamiltonians and they have similar physics of the superconductivity.

The discovery of superconductivity in the nickelate oxide [7] certainly has motivated the study of the effective Hamiltonian in this paper. However, two recent experimental papers reported nonsuperconductivity results in $\mathrm{Nd}_{1-x} \mathrm{Sr}_{x} \mathrm{NiO}_{2}$ [8,9], rendering the presence of superconductivity controversial. The existence of superconductivity in the doped nickelate oxides is a crucial issue and needs further experimental exploration. Our study here is the consequence of a quantumchemical description of $\mathrm{Nd}_{1-x} \mathrm{Sr}_{x} \mathrm{NiO}_{2}$; however, we cannot provide a direct theoretical implication of the existence of superconductivity. The effective Hamiltonian in this work provides essential support for, and constraint on, models to describe the low-energy physics of the nickelate oxides, regardless of the presence of superconductivity. In our recent experimental work [44] we performed Raman scattering on a $\mathrm{NdNiO}_{2}$ single crystal and measured the Heisenberg superexchange strength $J=25 \mathrm{meV}$ from the two-magnon peak, in good agreement with our present work. Although the current situation is not clear, we hope that our work will help us understand the electronic structure of nickelate oxides.

In conclusion, we have explicitly derived a single-band effective $t-t^{\prime}-J$ model Hamiltonian for Ni-O-based compounds starting from a three-band model based on the density functional theory.

Note added. Recently, we noticed related theoretical works for nickelates [45-52]. The pairing of hole carriers in the oxygen $p \pi$ orbitals is discussed in Ref. [47]. First-principles simulations within the GGA have been worked out in Refs. [45,46,49-52]. Dynamical mean field theory is carried out in Ref. [52] and random-phase approximation analysis of the pairing symmetry is done in Refs. [46,49]. The hopping parameters for the three-band Hubbard model are (implicitly or explicitly) given by the Wannier fitting in Refs. [45,46,49-
52], with values similar to those in our work. The hopping parameters $\left(t\right.$ and $t^{\prime}$ ) of the effective $\mathrm{Ni} 3 d_{x^{2}-y^{2}}$ band are also given in Refs. [45,49-51] with $t \sim 370 \mathrm{meV}$ and $t^{\prime} \sim$ $-100 \mathrm{meV}$, which are larger than the values $t=265 \mathrm{meV}$ and $t^{\prime}=-21 \mathrm{meV}$ renormalized by correlations in our work. The exchange interaction is estimated as $J=100 \mathrm{meV}$ in Ref. [49], larger than our value $J=28.6 \mathrm{meV}$. The HubbardMott scenario is also proposed in Ref. [48] and the chargetransfer gap is estimated as $\epsilon=7-9 \mathrm{eV}$, which is larger than our value of $\epsilon=4.2 \mathrm{eV}$. In the Ref. [48], the $S=1 \mathrm{Ni}^{2+}$ state is proposed when the hole is doped into the $\mathrm{NiO}_{2}$ planes, different from the $S=0 \mathrm{Ni}^{2+}$ state in our work. We note that if the charge-transfer gap is taken as the value we use in the work, $\epsilon=4.2 \mathrm{eV}$, the $S=0 \mathrm{Ni}^{2+}$ state is favored according to the calculation in Ref. [48]. There are many other related theoretical works [53-59].

\section{ACKNOWLEDGMENTS}

J.-W.M thanks J. S. Chen for help with drawing Fig. 1. J.-W.M was partially supported by the program for Guangdong Introducing Innovative and Entrepreneurial Teams (Grant No. 2017ZT07C062). This work was also supported by National Science Foundation of China (Grants No. 11774143 and No. 11774300), and the Shenzhen Fundamental Research Foundation (Grant No. JCYJ20170817105007999). The computational resources were provided by the Center for Computational Science and Engineering of Southern University of Science and Technology.

\section{APPENDIX A: SUPPLEMENTARY DFT RESULTS FOR $\mathrm{Nd}_{1-x} \mathrm{Sr}_{x} \mathrm{NiO}_{2}$}

In this Appendix we provide supplementary DFT results for $\mathrm{Nd}_{1-x} \mathrm{Sr}_{x} \mathrm{NiO}_{2}$. The main purpose of the supplementary 

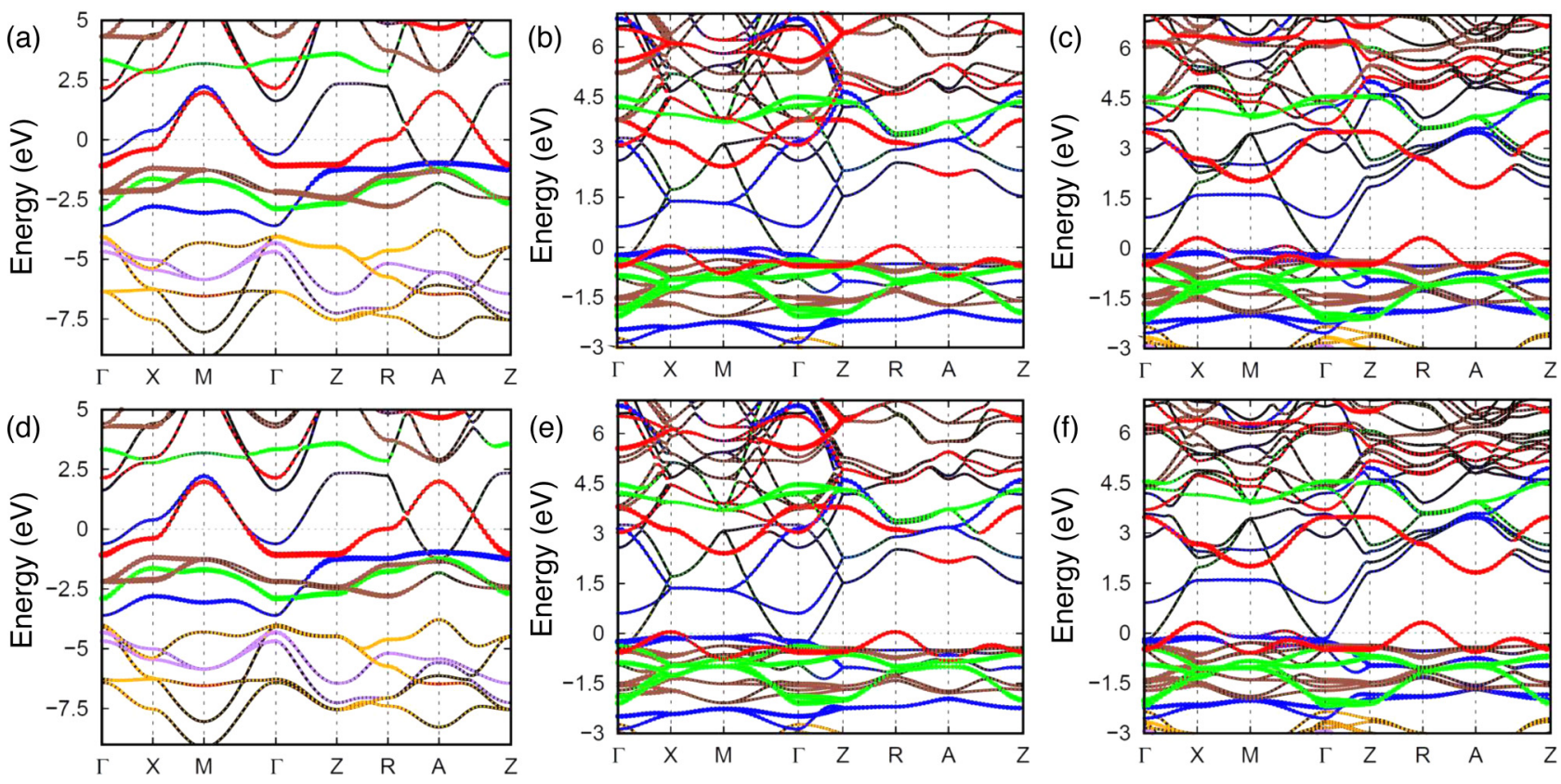

FIG. 9. Similar to Fig. 8, but for the SCAN band structures of $\mathrm{Nd}_{1-x} \mathrm{Sr}_{x} \mathrm{NiO}_{2}$.

results is twofold: (a) to check the validity of the $\mathrm{Nd}$ pseudopotential with the core-level $4 f$ electrons and (b) to compare the GGA and SCAN band structures to the HSE06 results in the main text. The spin-orbital coupling is also checked.

\section{GGA $+U$ calculations for the $\mathrm{Nd} 4 f$ valence electron pseudopotential}

Like $\mathrm{Nd}^{3+}$ in $\mathrm{Nd}_{2} \mathrm{CuO}_{4}$ [24] and $\mathrm{Ho}^{3+}$ in $\mathrm{HoNiO}_{3}$ [25], $\mathrm{Nd}^{3+}$ has the local $4 f$ moment far below the Fermi energy level $E_{F}$. In the main text we treated the $4 f$ electrons in $\mathrm{Nd}^{3+}$ as the core-level electrons in the $\mathrm{Nd}$ pseudopotential. In this section we verify the validity of this treatment.

Figure 7 shows the band structure of $G$-type AFM states for $\mathrm{NdNiO}_{2}$ and $\mathrm{Nd}_{0.75} \mathrm{Sr}_{0.25} \mathrm{NiO}_{2}$ calculated with the $4 f$ valence electron pseudopotential within the GGA $+U$ scheme. Without the $U$ term, the $4 f$ electrons form very localized bands near the Fermi level. In the $G$-type AFM states, the local moments of $4 f$ electrons are also AFM within the same $\mathrm{Nd}$ plane and between $\mathrm{Nd}$ planes along the $c$ direction. We take the on-site interaction for $4 f$ electrons to be $U_{4 f}=10 \mathrm{eV}$ in the GGA $+U$ calculation, which splits the $4 f$ bands above and below the Fermi energy level $E_{F}$. During the calculations, we also include the spin-orbital couplings.

According to Fig. 7, we can see that the $4 f$ electron states couple to Nd $5 d$ bands; however, this does not significantly change the band structures near the Fermi level. Thus we can treat $4 f$ orbitals as the core-level electrons in the $\mathrm{Nd}$ pseudopotential.

\section{GGA and SCAN band structures}

In this section we present the band structures for $\mathrm{Nd}_{1-x} \mathrm{Sr}_{x} \mathrm{NiO}_{2}$ within the GGA and SCAN functionals without and with spin-orbital couplings in Figs. 8 and 9, respectively. Again, once SOC is taken into account, there is no significant change in the band structures. The main features of SCAN band structures are very close to those in HSE06 band structures.

\section{APPENDIX B: HSE06 RESULTS OF LaNiO}

The LDA result for $\mathrm{LaNiO}_{2}$ has been studied previously [6]. In this Appendix we implement the HSE06 hybrid functional to calculated band structures of the nonmagnetic state
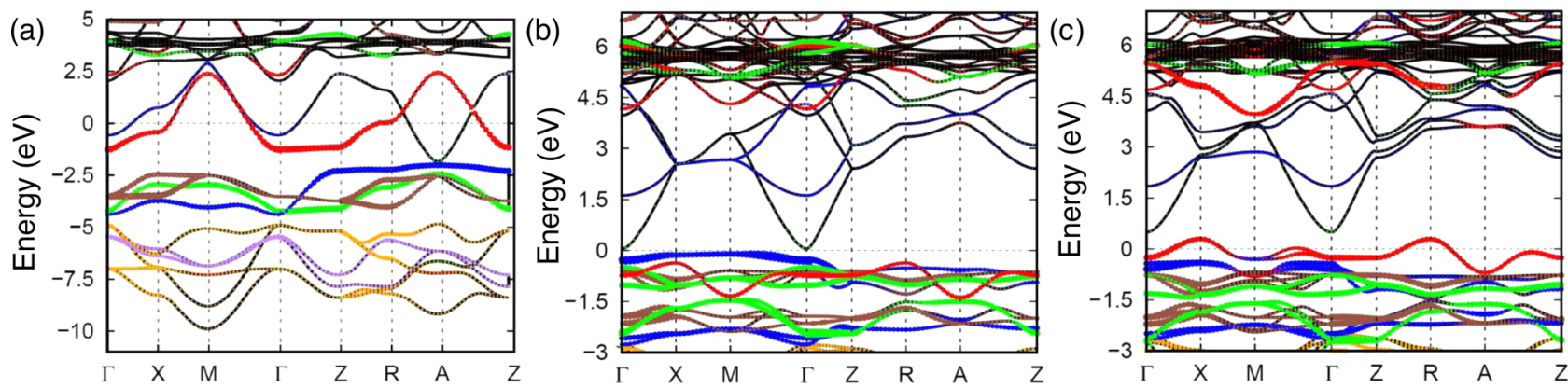

FIG. 10. HSE06 band structures of $\mathrm{La}_{1-x} \mathrm{Sr}_{x} \mathrm{NiO}_{2}$ without SOC: (a) nonmagnetic state for $\mathrm{LaNiO}_{2}$, (b) $G$-type $\mathrm{AFM}$ state for $\mathrm{LaNiO}$, and (c) $G$-type AFM state for $\mathrm{La}_{0.75} \mathrm{Sr}_{0.25} \mathrm{NiO}_{2}$, corresponding to Figs. 1(b), 3(a), and 3(b), respectively. 
in $\mathrm{LaNiO}_{2}$ and $G$-type AFM states for a supercell in $\mathrm{LaNiO}_{2}$ and $\mathrm{La}_{0.75} \mathrm{Sr}_{0.25} \mathrm{NiO}_{2}$ in Fig. 10. The band structures are very similar to the results of $\mathrm{NdNiO}_{2}$, implying the generic electronic structures in the $R \mathrm{NiO}_{2}$ family.
[1] J. G. Bednorz and K. A. Müller, Possible high $T_{c}$ superconductivity in the Ba-La-Cu-O system, Z. Phys. B 64, 189 (1986).

[2] P. A. Lee, N. Nagaosa, and X.-G. Wen, Doping a Mott insulator: Physics of high-temperature superconductivity, Rev. Mod. Phys. 78, 17 (2006).

[3] Y. Kamihara, T. Watanabe, M. Hirano, and H. Hosono, Iron-based layered superconductor $\mathrm{La}\left[\mathrm{O}_{1-x} \mathrm{~F}_{x}\right] \mathrm{FeAs}(x=$ 0.05-0.12) with $T_{c}=26 \mathrm{~K}$, J. Am. Chem. Soc. 130, 3296 (2008).

[4] H. Hosono and K. Kuroki, Iron-based superconductors: Current status of materials and pairing mechanism, Physica C 514, 399 (2015).

[5] V. I. Anisimov, D. Bukhvalov, and T. M. Rice, Electronic structure of possible nickelate analogs to the cuprates, Phys. Rev. B 59, 7901 (1999).

[6] K.-W. Lee and W. E. Pickett, Infinite-layer $\mathrm{LaNiO}_{2}: \mathrm{Ni}^{1+}$ is not $\mathrm{Cu}^{2+}$, Phys. Rev. B 70, 165109 (2004).

[7] D. Li, K. Lee, B. Y. Wang, M. Osada, S. Crossley, H. R. Lee, Y. Cui, Y. Hikita, and H. Y. Hwang, Superconductivity in an infinite-layer nickelate, Nature (London) 572, 624 (2019).

[8] Q. Li, C. He, J. Si, X. Zhu, Y. Zhang, and H.-H. Wen, Absence of superconductivity in bulk $\mathrm{Nd}_{1-x} \mathrm{Sr}_{x} \mathrm{NiO}_{2}(x=0.2,0.4)$, arXiv:1911.02420.

[9] X. Zhou, Z. Feng, P. Qin, H. Yan, S. Hu, H. Guo, X. Wang, H. Wu, X. Zhang, H. Chen, X. Qiu, and Z. Liu, Absence of superconductivity in $\mathrm{Nd}_{0.8} \mathrm{Sr}_{0.2} \mathrm{NiO}_{x}$ thin films without chemical reduction, arXiv:1911.04662.

[10] M. L. Medarde, Structural, magnetic and electronic properties of $\mathrm{RNiO}_{3}$ perovskites $(\mathrm{R}=$ rare earth), J. Phys.: Condens. Matter 9, 1679 (1997).

[11] G. Catalan, Progress in perovskite nickelate research, Phase Transit. 81, 729 (2008).

[12] S. Middey, J. Chakhalian, P. Mahadevan, J. W. Freeland, A. J. Millis, and D. D. Sarma, Physics of ultrathin films and heterostructures of rare-earth nickelates, Annu. Rev. Mater. Res. 46, 305 (2016).

[13] S. Catalano, M. Gibert, J. Fowlie, J. Íñiguez, J.-M. Triscone, and J. Kreisel, Rare-earth nickelates $\mathrm{RNiO}_{3}$ : Thin films and heterostructures, Rep. Prog. Phys. 81, 046501 (2018).

[14] M. Crespin, P. Levitz, and L. Gatineau, Reduced forms of $\mathrm{LaNiO}_{3}$ perovskite. Part 1.-Evidence for new phases: $\mathrm{La}_{2} \mathrm{Ni}_{2} \mathrm{O}_{5}$ and $\mathrm{LaNiO}_{2}$, J. Chem. Soc. Faraday Trans. 2 79, 1181 (1983).

[15] M. A. Hayward, M. A. Green, M. J. Rosseinsky, and J. Sloan, Sodium hydride as a powerful reducing agent for topotactic oxide deintercalation: Synthesis and characterization of the nickel(I) oxide $\mathrm{LaNiO}_{2}$, J. Am. Chem. Soc. 121, 8843 (1999).

[16] M. A. Hayward and M. J. Rosseinsky, Synthesis of the infinite layer $\mathrm{Ni}(\mathrm{I})$ phase $\mathrm{NdNiO}_{2+x}$ by low temperature reduction of $\mathrm{NdNiO}_{3}$ with sodium hydride, Solid State Sci. 5, 839 (2003).

[17] M. Kawai, S. Inoue, M. Mizumaki, N. Kawamura, N. Ichikawa, and Y. Shimakawa, Reversible changes of epitaxial thin films from perovskite $\mathrm{LaNiO}_{3}$ to infinite-layer structure $\mathrm{LaNiO}_{2}$, Appl. Phys. Lett. 94, 082102 (2009).
[18] D. Kaneko, K. Yamagishi, A. Tsukada, T. Manabe, and M. Naito, Synthesis of infinite-layer $\mathrm{LaNiO}_{2}$ films by metal organic decomposition, Physica C 469, 936 (2009).

[19] M. Kawai, K. Matsumoto, N. Ichikawa, M. Mizumaki, O. Sakata, N. Kawamura, S. Kimura, and Y. Shimakawa, Orientation change of an infinite-layer structure $\mathrm{LaNiO}_{2}$ epitaxial thin film by annealing with $\mathrm{CaH}_{2}$, Cryst. Growth Des. 10, 2044 (2010).

[20] A. Ikeda, Y. Krockenberger, H. Irie, M. Naito, and $H$. Yamamoto, Direct observation of infinite $\mathrm{NiO}_{2}$ planes in $\mathrm{LaNiO}_{2}$ films, Appl. Phys. Express 9, 061101 (2016).

[21] P. W. Anderson, The resonating valence bond state in $\mathrm{La}_{2} \mathrm{CuO}_{4}$ and superconductivity, Science 235, 1196 (1987).

[22] F. C. Zhang and T. M. Rice, Effective Hamiltonian for the superconducting Cu oxides, Phys. Rev. B 37, 3759 (1988).

[23] M. S. Hybertsen, E. B. Stechel, M. Schluter, and D. R. Jennison, Renormalization from density-functional theory to strong-coupling models for electronic states in $\mathrm{Cu}-\mathrm{O}$ materials, Phys. Rev. B 41, 11068 (1990).

[24] H. Casalta, P. Bourges, M. d'Astuto, D. Petitgrand, and A. Ivanov, Magnetic behavior of $\mathrm{Nd}$ in $\mathrm{Nd}_{2} \mathrm{CuO}_{4}$ above $1.5 \mathrm{~K}$, Phys. Rev. B 57, 471 (1998).

[25] M. T. Fernández-Díaz, J. A. Alonso, M. J. Martínez-Lope, M. T. Casais, and J. L. García-Muñoz, Magnetic structure of the $\mathrm{HoNiO}_{3}$ perovskite, Phys. Rev. B 64, 144417 (2001).

[26] M. Kawamura, K. Yoshimi, T. Misawa, Y. Yamaji, S. Todo, and N. Kawashima, Quantum lattice model solver $\mathcal{H} \phi$, Comput. Phys. Commun. 217, 180 (2017).

[27] F. Y. Bruno, K. Z. Rushchanskii, S. Valencia, Y. Dumont, C. Carrétéro, E. Jacquet, R. Abrudan, S. Blügel, M. Ležaić, M. Bibes, and A. Barthélémy, Rationalizing strain engineering effects in rare-earth nickelates, Phys. Rev. B 88, 195108 (2013).

[28] P. Fulde, V. Zevin, and G. Zwicknagl, Model for heavy-fermion behavior of $\mathrm{Nd}_{1.8} \mathrm{Ce}_{0.2} \mathrm{CuO}_{4}$, Z. Phys. B 92, 133 (1993).

[29] M. Hepting, D. Li, C. J. Jia, H. Lu, E. Paris, Y. Tseng, X. Feng, M. Osada, E. Been, Y. Hikita et al., Electronic structure of the parent compound of superconducting infinite-layer nickelates, [Nat. Mater. (to be published), (2020)], doi:10.1038/s41563019-0585-z.

[30] R. O. Jones, Density functional theory: Its origins, rise to prominence, and future, Rev. Mod. Phys. 87, 897 (2015).

[31] J. Heyd, G. E. Scuseria, and M. Ernzerhof, Hybrid functionals based on a screened Coulomb potential, J. Chem. Phys. 118, 8207 (2003).

[32] G. Kresse and J. Furthmüller, Efficient iterative schemes for $a b$ initio total-energy calculations using a plane-wave basis set, Phys. Rev. B 54, 11169 (1996).

[33] M. Gajdoš, K. Hummer, G. Kresse, J. Furthmüller, and F. Bechstedt, Linear optical properties in the projector-augmented wave methodology, Phys. Rev. B 73, 045112 (2006).

[34] J. Hafner, Ab-initio simulations of materials using VASP: Density-functional theory and beyond, J. Comput. Chem. 29, 2044 (2008). 
[35] J. P. Perdew, K. Burke, and M. Ernzerhof, Generalized Gradient Approximation Made Simple, Phys. Rev. Lett. 77, 3865 (1996).

[36] J. Sun, A. Ruzsinszky, and J. P. Perdew, Strongly Constrained and Appropriately Normed Semilocal Density Functional, Phys. Rev. Lett. 115, 036402 (2015).

[37] H. J. Monkhorst and J. D. Pack, Special points for Brillouinzone integrations, Phys. Rev. B 13, 5188 (1976).

[38] A. I. Liechtenstein, V. I. Anisimov, and J. Zaanen, Densityfunctional theory and strong interactions: Orbital ordering in Mott-Hubbard insulators, Phys. Rev. B 52, R5467 (1995).

[39] W. E. Pickett, Electronic structure of the high-temperature oxide superconductors, Rev. Mod. Phys. 61, 433 (1989).

[40] M.-Y. Choi, K.-W. Lee, and W. E. Pickett, Role of $4 f$ states in infinite-layer $\mathrm{NdNiO}_{2}$, Phys. Rev. B 101, 020503(R) (2020).

[41] A. A. Mostofi, J. R. Yates, Y.-S. Lee, I. Souza, D. Vanderbilt, and N. Marzari, wannier90: A tool for obtaining maximallylocalised Wannier functions, Comput. Phys. Commun. 178, 685 (2008).

[42] A. A. Mostofi, J. R. Yates, G. Pizzi, Y.-S. Lee, I. Souza, D. Vanderbilt, and N. Marzari, An updated version of wannier90: A tool for obtaining maximally-localised Wannier functions, Comput. Phys. Commun. 185, 2309 (2014).

[43] E. Dagotto, A. Nazarenko, and M. Boninsegni, Flat Quasiparticle Dispersion in the 2D $t-j$ Model, Phys. Rev. Lett. 73, 728 (1994).

[44] Y. Fu, L. Wang, H. Cheng, S. Pei, X. Zhou, J. Chen, S. Wang, R. Zhao, W. Jiang, C. Liu, M. Huang, X. Wang, Y. Zhao, D. Yu, S. Wang, and J.-W. Mei, Electronic structures and spin fluctuations in nickelate oxide $\mathrm{NdNiO}_{2}$, arXiv:1911.03177.

[45] A. S. Botana and M. R. Norman, Similarities and Differences Between Infinite-Layer Nickelates and Cuprates and Implications for Superconductivity, Phys. Rev. X 10, 011024 (2020).

[46] H. Sakakibara, H. Usui, K. Suzuki, T. Kotani, H. Aoki, and K. Kuroki, Model construction and a possibility of cuprate-like pairing in a new d9 nickelate superconductor $(\mathrm{Nd}, \mathrm{Sr}) \mathrm{NiO}_{2}$, arXiv:1909.00060.
[47] J. E. Hirsch and F. Marsiglio, Hole superconductivity in infinitelayer nickelates, Physica C 566, 1353534 (2009).

[48] M. Jiang, M. Berciu, and G. A. Sawatzky, Doped holes in $\mathrm{NdNiO}_{2}$ and high- $T_{c}$ cuprates show little similarity, arXiv:1909.02557.

[49] X. Wu, D. D. Sante, T. Schwemmer, W. Hanke, H. Y. Hwang, S. Raghu, and R. Thomale, Robust $d_{x^{2}-y^{2}}$-wave superconductivity of infinite-layer nickelates, arXiv:1909.03015.

[50] Y. Nomura, M. Hirayama, T. Tadano, Y. Yoshimoto, K. Nakamura, and R. Arita, Formation of 2D single-component correlated electron system and band engineering in the nickelate superconductor $\mathrm{NdNiO}_{2}$, Phys. Rev. B 100, 205138 (2019).

[51] J. Gao, Z. Wang, C. Fang, and H. Weng, Electronic structures and topological properties in nickelates $L n_{n+1} \mathrm{Ni}_{n} \mathrm{O}_{2 n+2}$, arXiv:1909.04657.

[52] S. Ryee, H. Yoon, T. J. Kim, M. Y. Jeong, and M. J. Han, Induced magnetic two-dimensionality by hole doping in superconducting $\mathrm{Nd}_{1-x} \mathrm{Sr}_{x} \mathrm{NiO}_{2}$, arXiv:1909.05824.

[53] G.-M. Zhang, Y.-F. Yang, and F.-C. Zhang, Self-doped Mott insulator for parent compounds of nickelate superconductors, Phys. Rev. B 101, 020501(R) (2020).

[54] Y.-H. Zhang and A. Vishwanath, Type II $t$ - $j$ model in superconducting nickelate $\mathrm{Nd}_{1-x} \mathrm{Sr}_{x} \mathrm{NiO}_{2}$, arXiv:1909.12865.

[55] P. Jiang, L. Si, Z. Liao, and Z. Zhong, Electronic structure of rare-earth infinite-layer $R \mathrm{NiO}_{2}(R=\mathrm{La}, \mathrm{Nd})$, Phys. Rev. B 100, 201106(R) (2019).

[56] L.-H. Hu and C. Wu, Two-band model for magnetism and superconductivity in nickelates, Phys. Rev. Research 1, 032046(R) (2019).

[57] J. Chang, J. Zhao, and Y. Ding, Hund-Heisenberg model in superconducting infinite-layer nickelates, arXiv:1911.12731.

[58] P. Werner and S. Hoshino, Nickelate superconductors: Multiorbital nature and spin freezing, Phys. Rev. B 101, 041104(R) (2020).

[59] Z. Liu, Z. Ren, W. Zhu, Z. F. Wang, and J. Yang, Electronic and magnetic structure of infinite-layer $\mathrm{NdNiO}_{2}$ : Trace of antiferromagnetic metal, arXiv:1912.01332. 